CRYSTALLOGRAPHIC COMMUNICATIONS

ISSN 2056-9890

Received 3 August 2020

Accepted 8 September 2020

Edited by H. Ishida, Okayama University, Japan

Keywords: crystal structure; melaminium cation; cyanoacetate anion; hydrogen bonding.

CCDC reference: 2030784

Supporting information: this article has supporting information at journals.iucr.org/e

\section{Crystal structure of melaminium cyanoacetate monohydrate}

\author{
Bhawani Sigdel Regmi, ${ }^{a *}$ Allen Apblett ${ }^{\mathrm{b}}$ and Douglas Powell ${ }^{\mathrm{c}}$
}

\begin{abstract}
${ }^{\mathbf{a}}$ Department of Chemistry and Biochemistry, University of North Georgia, Oakwood, Georgia, USA, ${ }^{\mathbf{b}}$ Oklahoma State University, Stillwater, Oklahoma, USA, and ' ${ }^{c}$ University of Oklahoma, Norman, Oklahoma, USA. *Correspondence e-mail: bsigdelregmi@ung.edu
\end{abstract}

The asymmetric unit of the title compound, 2,4,6-triamino-1,3,5-triazin-1-ium cyanoacetate monohydrate, $\mathrm{C}_{3} \mathrm{H}_{7} \mathrm{~N}_{6}{ }^{+} \cdot \mathrm{NCCH}_{2} \mathrm{COO}^{-} \cdot \mathrm{H}_{2} \mathrm{O}$, consists of a melaminium cation, a cyanoacetate anion and a water molecule, which are connected to each other via $\mathrm{N}-\mathrm{H} \cdots \mathrm{O}$ and $\mathrm{O}-\mathrm{H} \cdots \mathrm{O}$ hydrogen bonds, generating an eight-membered ring. In the crystal, the melaminium cations are connected by two pairs of $\mathrm{N}-\mathrm{H} \cdots \mathrm{N}$ hydrogen bonds, forming tapes along [110]. These tapes develop a three-dimensional network through $\mathrm{N}-\mathrm{H} \cdots \mathrm{O}, \mathrm{O}-\mathrm{H} \cdots \mathrm{O}, \mathrm{N}-$ $\mathrm{H} \cdots \mathrm{N}$ and $\mathrm{C}-\mathrm{H} \cdots \mathrm{O}$ hydrogen bonds between the cations, anions and water molecules.

\section{Chemical context}

Melamine (systematic name: 2,4,6-triamino-1,3,5-triazine), a trimer of cyanamide, has many industrial applications. The cross-linked resins of melamine with formaldehyde have applications in adhesive coatings, laminations and flame retardants (Billmeyer, 1984). In the past, various organic melamine salts were tested as potential melamine substitutes for melamine urea formaldehyde resins (Weinstabl et al., 2001). In general, protonation of melamine with organic and inorganic acids has been found to yield compounds with extensive hydrogen-bonding networks involving both $\mathrm{N}-$ $\mathrm{H} \cdots \mathrm{O}$ and $\mathrm{O}-\mathrm{H} \cdots \mathrm{O}$ hydrogen bonds. This paper is a part of our investigation of the chemistry of cyanoacetate with nitrogen-based cations and their potential application as flame retardants since cyanoacetic acid is an analogue to polyacrylonitrile. It is well known that polyacrylonitrile is used in industry to manufacture carbon fibers because of its ability to produce carbon char (Bacon \& Hoses, 1986). Cyanoacetic acid has a nitrile group and also can act as acid source, both of which could enhance the flame-retarding properties.<smiles>N#CCC(=O)OCCO</smiles>

\section{Structural commentary}

The asymmetric unit of the title compound consists of a melaminium cation, a cyanoacetate anion and a water molecule, which are connected to each other via $\mathrm{N}-\mathrm{H} \cdots \mathrm{O}$ and 
$\mathrm{O}-\mathrm{H} \cdots \mathrm{O}$ hydrogen bonds, generating an eight-membered ring (Fig. 1). The six-membered ring of the melaminium cation shows significant distortion from a hexagonal shape. The bond distances $[\mathrm{C}-\mathrm{N}=1.322(2)-1.368(2) \AA]$ and the angles $[\mathrm{C}-$ $\mathrm{N}-\mathrm{C}=115.76(15)-119.08(14)^{\circ}$ and $\mathrm{N}-\mathrm{C}-\mathrm{N}=121.44(15)-$ $125.42(15)^{\circ}$ ] fall within similar ranges to those reported for similar singly protonated melaminium salts of simple alkyl mono- and dicarboxylic acids, namely, melaminium acetate acetic acid solvate (Perpétuo \& Janczak, 2002), melaminium maleate (Janczak \& Perpétuo, 2004), melaminium formate (Perpétuo et al., 2005), melaminium tartarate (Su et al., 2009), bis(melaminium) succinate (Froschauer \& Weil, 2012a) and melaminium hydrogen malonate (Froschauer \& Weil, 2012b). On the other hand, the angles in the six-membered ring of unprotonated melamine (Adam et al., 2010) are in the range 124.86 (17) to $125.51(17)^{\circ}$.

In the anion, both $\mathrm{O}$ atoms of the carboxylate group are involved in hydrogen bonds to amino groups of adjacent melaminium ions. The nitrile group has a bond length of 1.145 (2) $\AA$ that is typical of a nitrile (Kanters et al., 1978). The angle at the nitrile carbon, $\mathrm{N} \equiv \mathrm{C}-\mathrm{C}$, is $179.30(19)^{\circ}$ which is close to the theoretical value of $180^{\circ}$. The $\mathrm{O}$ atom of the water molecule acts as a lone-pair donor to the protonated nitrogen

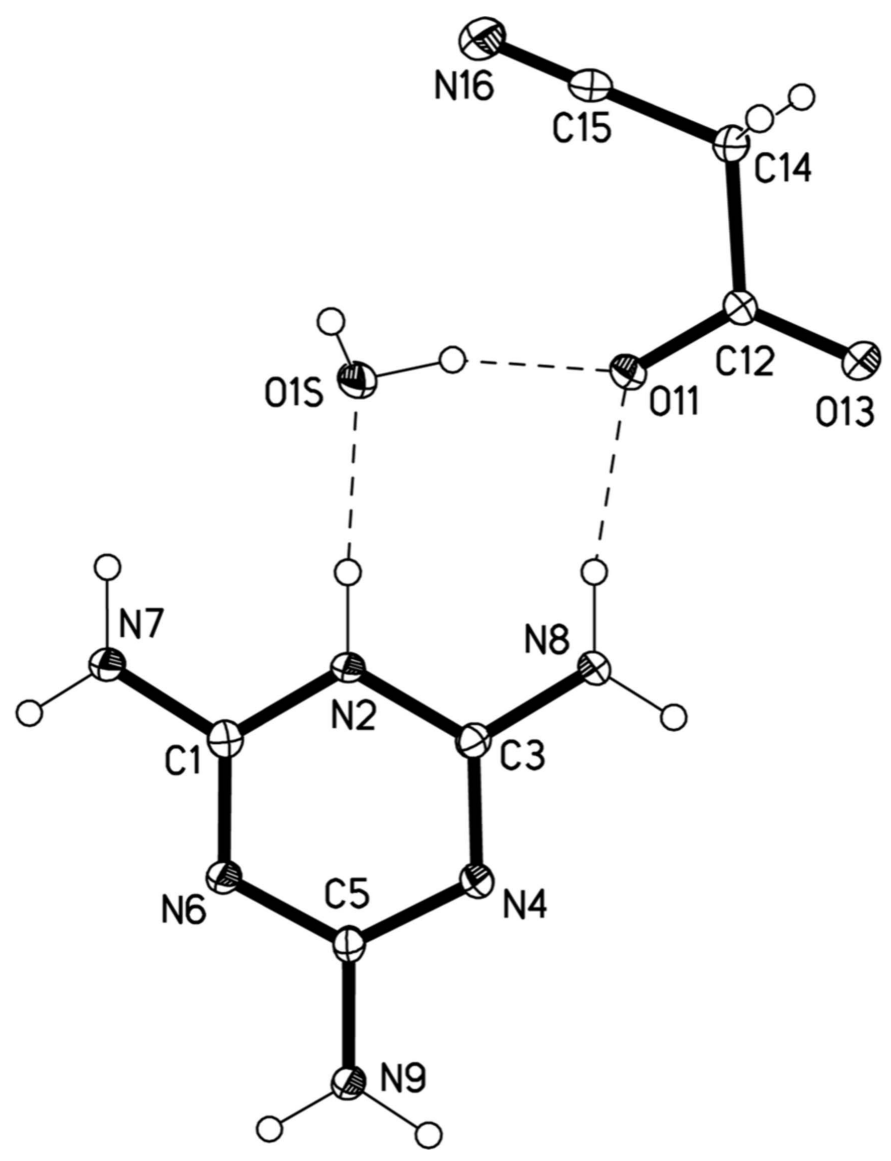

Figure 1

Molecular structure of the title compound, showing $50 \%$ probability displacement ellipsoids and the atom-numbering scheme. Hydrogen atoms are shown as spheres of arbitrary radius and hydrogen bonds as dashed lines.
Table 1

Hydrogen-bond geometry $\left(\AA{ }^{\circ}\right)$.

\begin{tabular}{|c|c|c|c|c|}
\hline$D-\mathrm{H} \cdots A$ & $D-\mathrm{H}$ & $\mathrm{H} \cdots A$ & $D \cdots A$ & $D-\mathrm{H} \cdots A$ \\
\hline $\mathrm{J} 2-\mathrm{H} 2 \cdots \mathrm{O} 1 S$ & $0.90(2)$ & $1.81(2)$ & $2.7067(19)$ & $176.4(19)$ \\
\hline $\mathrm{N} 7-\mathrm{H} 7 A \cdots \mathrm{O} 13^{\mathrm{i}}$ & $0.89(2)$ & $2.00(2)$ & $2.881(2)$ & $168(2)$ \\
\hline $\mathrm{N} 7-\mathrm{H} 7 B \cdots \mathrm{N} 16^{\mathrm{ii}}$ & $0.92(2)$ & $2.13(2)$ & 3.001 (2) & $155.6(18)$ \\
\hline $\mathrm{N} 8-\mathrm{H} 8 A \cdots \mathrm{O} 11$ & $0.91(2)$ & $2.01(2)$ & $2.891(2)$ & $164.6(19)$ \\
\hline $\mathrm{N} 8-\mathrm{H} 8 B \cdots \mathrm{N} 4^{\mathrm{iii}}$ & $0.88(2)$ & $2.07(2)$ & $.952(2)$ & $176(2)$ \\
\hline $\mathrm{N} 9-\mathrm{H} 9 A \cdots \mathrm{O} 13^{\mathrm{iii}}$ & $90(2)$ & $2.08(2)$ & 2.792 & 135.7 (19) \\
\hline $\mathrm{N} 9-\mathrm{H} 9 B \cdots \mathrm{N} 6^{\mathrm{iv}}$ & $0.90(2)$ & $2.08(2)$ & $2.980(2)$ & $174(2)$ \\
\hline $\mathrm{C} 14-\mathrm{H} 14 B \cdots \mathrm{O} 1 S^{\mathrm{v}}$ & 0.99 & 2.46 & $3.233(2)$ & 134 \\
\hline $\mathrm{O} 1 S-\mathrm{H} 1 S A \cdots \mathrm{O} 11$ & $0.93(2)$ & $1.78(2)$ & $2.6860(19)$ & $163.3(19)$ \\
\hline $\mathrm{O} 1 S-\mathrm{H} 1 S B \cdots \mathrm{O} 11^{\mathrm{vi}}$ & $0.87(2)$ & $1.97(2)$ & 2.8351 (19) & $178(2)$ \\
\hline
\end{tabular}

Symmetry codes: (i) $x-1, y-1, z$; (ii) $-x, y-\frac{1}{2},-z+\frac{1}{2}$; (iii) $-x+2,-y+1,-z+1$; (iv) $-x+1,-y,-z+1$; (v) $-x+1, y+\frac{1}{2},-z+\frac{1}{2}$; (vi) $x-1, y, z$.

of the melaminium ion that is present in the same eightmembered ring. The presence of the water molecule in the structure of melaminium cyanoacetate can be expected to contribute to fire retardancy as its release and evaporation will provide cooling.

\section{Supramolecular features}

The melaminium cation in the crystal is involved in altogether nine hydrogen bonds: for each melaminium cation, seven of them are of the hydrogen-bond donor type while the remaining two are of the acceptor type (Table 1). Neighbouring cations are connected by two pairs of $\mathrm{N}-\mathrm{H} \cdots \mathrm{N}$ hydrogen bonds $\left(\mathrm{N} 8-\mathrm{H} 8 B \cdots \mathrm{N} 4^{\mathrm{iii}}\right.$ and $\mathrm{N} 9-\mathrm{H} 9 B \cdots \mathrm{N} 6{ }^{\mathrm{iv}}$;

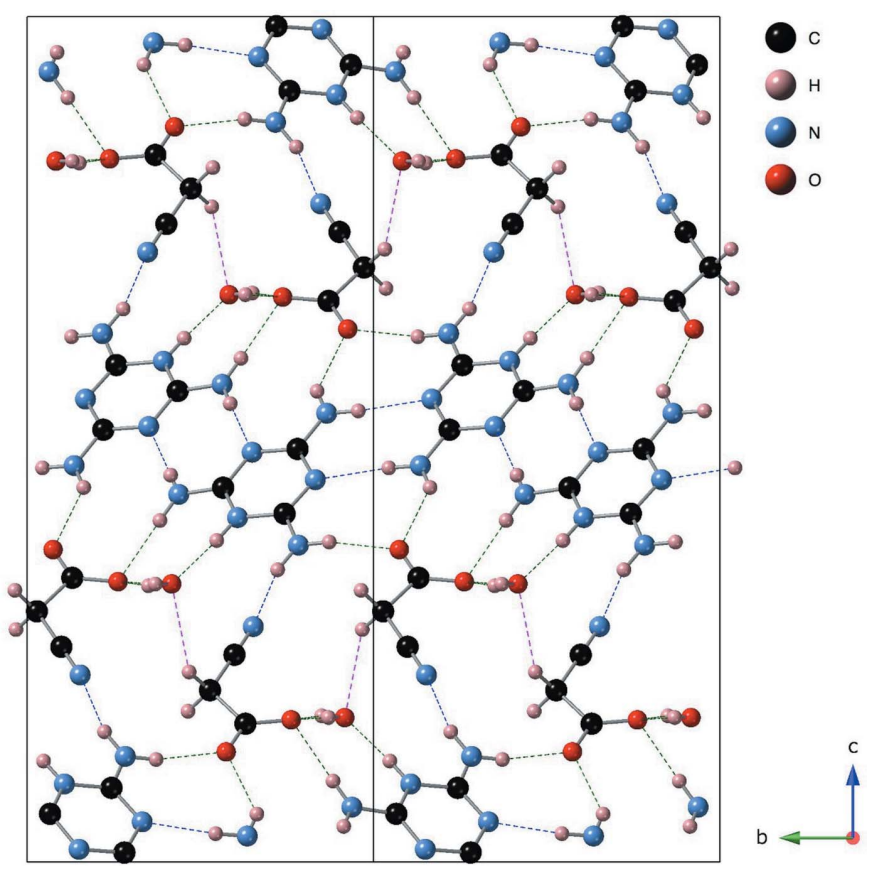

Figure 2

A packing diagram of the title compound, viewed down the $a$ axis, showing the $\mathrm{O}-\mathrm{H} \cdots \mathrm{O}$ and $\mathrm{N}-\mathrm{H} \cdots \mathrm{O}$ hydrogen bonds (green dashed lines), the $\mathrm{N}-\mathrm{H} \cdots \mathrm{N}$ hydrogen bonds (blue dashed lines) and the $\mathrm{C}-$ $\mathrm{H} \cdots \mathrm{O}$ hydrogen bonds (magenta dashed lines). 
symmetry codes as in Table 1) to form a tape-like structure propagating along [110] and running between the cyanoacetate anions. Three $\mathrm{N}-\mathrm{H} \cdots \mathrm{O}$ hydrogen bonds (N7$\mathrm{H} 7 A \cdots \mathrm{O} 13^{\mathrm{i}}, \quad \mathrm{N} 8-\mathrm{H} 8 A \cdots \mathrm{O} 11$ and $\mathrm{N} 9-\mathrm{H} 9 A \cdots \mathrm{O} 13^{\mathrm{iii}}$; Table 1) link the cation with three different cyanoacetate anions. Furthermore, the cation is also connected with a water molecule via an $\mathrm{N}-\mathrm{H} \cdots \mathrm{O}$ hydrogen bond $(\mathrm{N} 2-\mathrm{H} 2 \cdots \mathrm{O} 1 S)$ between the protonated imine and the water $\mathrm{O}$ atom. Finally, the cation is linked with the nitrile group of the anion via an $\mathrm{N}-\mathrm{H} \cdots \mathrm{N}$ hydrogen bond $\left(\mathrm{N} 7-\mathrm{H} 7 B \cdots \mathrm{N} 16^{\mathrm{ii}}\right.$; Table 1$)$. There also exist $\mathrm{O}-\mathrm{H} \cdots \mathrm{O} \quad(\mathrm{O} 1 S-\mathrm{H} 1 S A \cdots \mathrm{O} 11$ and $\mathrm{O} 1 \mathrm{~S}-$ $\mathrm{H} 1 S B \cdots \mathrm{O} 11^{\mathrm{vi}}$ ) hydrogen bonds between the water molecule and the anion. In addition, a $\mathrm{C}-\mathrm{H} \cdots \mathrm{O}$ hydrogen bond between the methylene $\mathrm{H}$ and water $\mathrm{O}$ atoms is observed as the $\mathrm{C}-\mathrm{H}$ group is activated because of the electron-withdrawing cyano group adjacent to it. Altogether, these hydrogen bonds existing between the cations, anions and water molecules generate a three-dimensional network (Fig. 2).

\section{Database survey}

A search of the Cambridge Structural Database (Version 5.40, update of May 2020; Groom et al., 2016) for 2,4,6-triamino1,3,5-triazin-1-ium showed more than 30 records; however, for 2,4,6-triamino-1,3,5-triazin-1-ium forming only single protonated salts with purely organic aliphatic carboxylic acids the search gave the following crystal structures: melamine with maleic acid (refcode ARUDAS; Janczak \& Perpétuo, 2004), with formic acid (FONMEB; Perpétuo et al., 2005), with acetic acid (EFAZOA; Perpétuo \& Janczak, 2002), with malonic acid (HOWRIV01; Froschauer \& Weil, 2012b), with succinic acid (LEGZEE; Froschauer \& Weil, 2012a), with nitrilotriacetic acid (MIHYAF; Hoxha et al., 2013) and with tartaric acid (VORSUR; Su et al., 2009). A search for organic co-crystals/ salts of cyanoacetic acid gave one structure, 4,4'-bipyridine bis(cyanoacetic acid) (Song et al., 2008). For metal complexes with cyanoacetic acid or cyanoacetate, 24 structures were reported, such as silver cyanoacetate (Edwards et al., 1997) and cadmium cyanoacetate (Post \& Trotter, 1974). In these metal salts, the metal is coordinated by the acetate group as well as the cyano group.

\section{Synthesis and crystallization}

A solution of cyanoacetic acid $(1.7 \mathrm{~g}, 20 \mathrm{mmol})$ in $100 \mathrm{ml}$ of deionized water was added to a solution of melamine $(2.5 \mathrm{~g}$, $20 \mathrm{mmol}$ ) in $100 \mathrm{ml}$ of deionized water. The reaction mixture was heated to $353 \mathrm{~K}$ for $3 \mathrm{~h}$. The resulting clear solution was cooled to room temperature and then was allowed to slowly evaporate. Single crystals of the title compound formed after several days.

\section{Refinement}

Crystal data, data collection and structure refinement details are summarized in Table 2. C-bound $\mathrm{H}$ atoms were initially
Table 2

Experimental details.

\begin{tabular}{|c|c|}
\hline Crystal data & \\
\hline Chemical formula & $\mathrm{C}_{3} \mathrm{H}_{7} \mathrm{~N}_{6}{ }^{+} \cdot \mathrm{C}_{3} \mathrm{H}_{2} \mathrm{NO}_{2}{ }^{-} \cdot \mathrm{H}_{2} \mathrm{O}$ \\
\hline$M_{\mathrm{r}}$ & 229.22 \\
\hline Crystal system, space group & Monoclinic, $P 2_{1} / c$ \\
\hline Temperature $(\mathrm{K})$ & 100 \\
\hline$a, b, c(\AA)$ & 4.6928 (6), $9.3881(13), 22.918$ (3) \\
\hline$\beta\left(^{\circ}\right)$ & $91.646(3)$ \\
\hline$V\left(\AA^{3}\right)$ & $1009.3(2)$ \\
\hline$Z$ & 4 \\
\hline Radiation type & Мо $K \alpha$ \\
\hline$\mu\left(\mathrm{mm}^{-1}\right)$ & 0.12 \\
\hline Crystal size $(\mathrm{mm})$ & $0.44 \times 0.17 \times 0.04$ \\
\hline Data collection & \\
\hline Diffractometer & Bruker $A P E X \mathrm{CCD}$ \\
\hline Absorption correction & $\begin{array}{l}\text { Multi-scan (SADABS; Bruker, } \\
\text { 2007) }\end{array}$ \\
\hline$T_{\min }, T_{\max }$ & $0.948,0.995$ \\
\hline $\begin{array}{l}\text { No. of measured, independent and } \\
\text { observed }[I>2 \sigma(I)] \text { reflections }\end{array}$ & $12615,2517,1856$ \\
\hline$R_{\text {int }}$ & 0.058 \\
\hline$(\sin \theta / \lambda)_{\max }\left(\AA^{-1}\right)$ & 0.668 \\
\hline Refinement & \\
\hline$R\left[F^{2}>2 \sigma\left(F^{2}\right)\right], w R\left(F^{2}\right), S$ & $0.047,0.126,1.00$ \\
\hline No. of reflections & 2517 \\
\hline No. of parameters & 172 \\
\hline $\mathrm{H}$-atom treatment & $\begin{array}{l}\mathrm{H} \text { atoms treated by a mixture of } \\
\text { independent and constrained } \\
\text { refinement }\end{array}$ \\
\hline$\Delta \rho_{\max }, \Delta \rho_{\min }\left(\mathrm{e} \AA^{-3}\right)$ & $0.28,-0.29$ \\
\hline
\end{tabular}

Computer programs: SMART and SAINT (Bruker, 2007), SHELXT (Sheldrick, 2015a), SHELXL2018/3 (Sheldrick, 2015b), SHELXTL (Sheldrick, 2008) and CrystalMaker (Palmer, 2014).

determined by geometry $(\mathrm{C}-\mathrm{H}=0.99 \AA)$ and were refined using a riding model, with $U_{\text {iso }}(\mathrm{H})=1.2 U_{\text {eq }}(\mathrm{C})$. $\mathrm{H}$ atoms bonded to $\mathrm{N}$ and $\mathrm{O}$ were located in a difference map, and their positions were refined freely, with $U_{\text {iso }}(\mathrm{H})=1.2 U_{\text {eq }}(\mathrm{N}$ or $\mathrm{O})$.

\section{References}

Adam, F., Lin, S. K., Quah, C. K., Hemamalini, M. \& Fun, H.-K. (2010). Acta Cryst. E66, o3033-03034.

Bacon, R. \& Hoses, T. N. (1986). High Performance Polymers, Their Origin and Development, edited by R. B. Saymour and G. S. Kirshambaum, p .342. New York/Amsterdam/London: Elsevier.

Billmeyer, F. W. (1984). Science, 3rd ed. New York: WileyInterscience.

Bruker (2007). SMART, SAINT and SADABS. Bruker AXS Inc., Madison, Wisconsin, USA.

Edwards, D. A., Mahon, M. F. \& Paget, T. J. (1997). Polyhedron, 16, 25-31.

Froschauer, B. \& Weil, M. (2012a). Acta Cryst. E68, o2553-o2554.

Froschauer, B. \& Weil, M. (2012b). Acta Cryst. E68, o2555-02555.

Groom, C. R., Bruno, I. J., Lightfoot, M. P. \& Ward, S. C. (2016). Acta Cryst. B72, 171-179.

Hoxha, K. \& Prior, T. J. (2013). Acta Cryst. E69, o1674-o1675.

Janczak, J. \& Perpétuo, G. J. (2004). Acta Cryst. C60, o211-o214.

Kanters, J. A., Roelofsen, G. \& Straver, L. H. (1978). Acta Cryst. B34, 1393-1395.

Palmer, D. C. (2014). CrystalMaker. CrystalMaker Software Ltd, Begbroke, England.

Perpétuo, G. J. \& Janczak, J. (2002). Acta Cryst. C58, o112-o114.

Perpétuo, G. J., Ribeiro, M. A. \& Janczak, J. (2005). Acta Cryst. E61, o1818-o1820. 
Post, M. L. \& Trotter, J. (1974). J. Chem. Soc. Dalton Trans. pp. 285288.

Sheldrick, G. M. (2008). Acta Cryst. A64, 112-122.

Sheldrick, G. M. (2015a). Acta Cryst. A71, 3-8.

Sheldrick, G. M. (2015b). Acta Cryst. C71, 3-8.
Song, G., Hao, E.-J. \& Li, W. (2008). Acta Cryst. E64, o2058.

Su, H., Lv, Y.-K. \& Feng, Y.-L. (2009). Acta Cryst. E65, o933o933.

Weinstabl, A., Binder, W. H., Gruber, H. \& Kantner, W. (2001). J. Appl. Polym. Sci. 81, 1654-1661. 


\section{supporting information}

Acta Cryst. (2020). E76, 1645-1648 [https://doi.org/10.1107/S2056989020012335]

\section{Crystal structure of melaminium cyanoacetate monohydrate}

\section{Bhawani Sigdel Regmi, Allen Apblett and Douglas Powell}

Computing details

Data collection: SMART (Bruker, 2007); cell refinement: SAINT (Bruker, 2007); data reduction: SAINT (Bruker, 2007); program(s) used to solve structure: SHELXT (Sheldrick, 2015a); program(s) used to refine structure: SHELXL2018/3 (Sheldrick, 2015b); molecular graphics: SHELXTL (Sheldrick, 2008) and CrystalMaker (Palmer, 2014); software used to prepare material for publication: SHELXTL (Sheldrick, 2008).

2,4,6-Triamino-1,3,5-triazin-1-ium cyanoacetate monohydrate

Crystal data

$\mathrm{C}_{3} \mathrm{H}_{7} \mathrm{~N}_{6}{ }^{+} \cdot \mathrm{C}_{3} \mathrm{H}_{2} \mathrm{NO}_{2}^{-} \cdot \mathrm{H}_{2} \mathrm{O}$

$M_{r}=229.22$

Monoclinic, $P 2{ }_{1} / c$

$a=4.6928(6) \AA$

$b=9.3881(13) \AA$

$c=22.918(3) \AA$

$\beta=91.646(3)^{\circ}$

$V=1009.3(2) \AA^{3}$

$Z=4$

Data collection

Bruker APEX CCD

diffractometer

$\varphi$ and $\omega$ scans

Absorption correction: multi-scan

(SADABS; Bruker, 2007)

$T_{\min }=0.948, T_{\max }=0.995$

12615 measured reflections

\section{Refinement}

Refinement on $F^{2}$

Least-squares matrix: full

$R\left[F^{2}>2 \sigma\left(F^{2}\right)\right]=0.047$

$w R\left(F^{2}\right)=0.126$

$S=1.00$

2517 reflections

172 parameters

0 restraints

Primary atom site location: structure-invariant direct methods
$F(000)=480$

$D_{\mathrm{x}}=1.509 \mathrm{Mg} \mathrm{m}^{-3}$

Mo $K \alpha$ radiation, $\lambda=0.71073 \AA$

Cell parameters from 3720 reflections

$\theta=2.3-28.1^{\circ}$

$\mu=0.12 \mathrm{~mm}^{-1}$

$T=100 \mathrm{~K}$

Needle, colourless

$0.44 \times 0.17 \times 0.04 \mathrm{~mm}$

2517 independent reflections 1856 reflections with $I>2 \sigma(I)$

$R_{\text {int }}=0.058$

$\theta_{\text {max }}=28.4^{\circ}, \theta_{\min }=1.8^{\circ}$

$h=-5 \rightarrow 6$

$k=-12 \rightarrow 12$

$l=-30 \rightarrow 30$

Secondary atom site location: difference Fourier map

Hydrogen site location: mixed

$\mathrm{H}$ atoms treated by a mixture of independent and constrained refinement

$w=1 /\left[\sigma^{2}\left(F_{\mathrm{o}}^{2}\right)+(0.064 P)^{2}+0.320 P\right]$

where $P=\left(F_{\mathrm{o}}{ }^{2}+2 F_{\mathrm{c}}{ }^{2}\right) / 3$

$(\Delta / \sigma)_{\max }=0.001$

$\Delta \rho_{\max }=0.28 \mathrm{e} \AA^{-3}$

$\Delta \rho_{\min }=-0.29$ e $\AA^{-3}$ 


\section{Special details}

Geometry. All esds (except the esd in the dihedral angle between two 1.s. planes) are estimated using the full covariance matrix. The cell esds are taken into account individually in the estimation of esds in distances, angles and torsion angles; correlations between esds in cell parameters are only used when they are defined by crystal symmetry. An approximate (isotropic) treatment of cell esds is used for estimating esds involving l.s. planes.

Fractional atomic coordinates and isotropic or equivalent isotropic displacement parameters $\left(\AA^{2}\right)$

\begin{tabular}{lllll}
\hline & $x$ & $y$ & $z$ & $U_{\text {iso }} / U_{\text {eq }}$ \\
\hline C1 & $0.3297(4)$ & $0.25701(18)$ & $0.41214(7)$ & $0.0117(4)$ \\
N2 & $0.4321(3)$ & $0.39286(15)$ & $0.40750(6)$ & $0.0121(3)$ \\
H2 & $0.359(4)$ & $0.453(2)$ & $0.3804(9)$ & $0.014^{*}$ \\
C3 & $0.6562(4)$ & $0.43434(17)$ & $0.44283(7)$ & $0.0111(4)$ \\
N4 & $0.7614(3)$ & $0.35064(14)$ & $0.48477(6)$ & $0.0126(3)$ \\
C5 & $0.6396(4)$ & $0.21954(17)$ & $0.48861(8)$ & $0.0125(4)$ \\
N6 & $0.4316(3)$ & $0.16816(15)$ & $0.45231(6)$ & $0.0135(3)$ \\
N7 & $0.1226(3)$ & $0.21696(16)$ & $0.37493(7)$ & $0.0151(3)$ \\
H7A & $0.059(4)$ & $0.128(2)$ & $0.3786(9)$ & $0.018^{*}$ \\
H7B & $0.055(4)$ & $0.277(2)$ & $0.3458(9)$ & $0.018^{*}$ \\
N8 & $0.7672(3)$ & $0.56251(15)$ & $0.43490(7)$ & $0.0137(3)$ \\
H8A & $0.711(4)$ & $0.617(2)$ & $0.4041(9)$ & $0.016^{*}$ \\
H8B & $0.914(5)$ & $0.586(2)$ & $0.4577(9)$ & $0.016^{*}$ \\
N9 & $0.7332(4)$ & $0.13535(16)$ & $0.53094(7)$ & $0.0202(4)$ \\
H9A & $0.870(5)$ & $0.163(2)$ & $0.5569(10)$ & $0.024^{*}$ \\
H9B & $0.673(5)$ & $0.044(3)$ & $0.5337(9)$ & $0.024^{*}$ \\
O11 & $0.6998(3)$ & $0.73778(13)$ & $0.33172(5)$ & $0.0157(3)$ \\
C12 & $0.7511(4)$ & $0.87001(18)$ & $0.33632(8)$ & $0.0129(4)$ \\
O13 & $0.9314(3)$ & $0.92554(14)$ & $0.36958(6)$ & $0.0224(3)$ \\
C14 & $0.5789(4)$ & $0.97232(18)$ & $0.29669(8)$ & $0.0163(4)$ \\
H14A & 0.465226 & 1.035861 & 0.321467 & $0.020^{*}$ \\
H14B & 0.713248 & 1.032642 & 0.275142 & $0.020^{*}$ \\
C15 & $0.3874(4)$ & $0.90159(18)$ & $0.25472(8)$ & $0.0156(4)$ \\
N16 & $0.2366(4)$ & $0.84731(17)$ & $0.22144(7)$ & $0.0237(4)$ \\
O1S & $0.2181(3)$ & $0.58309(13)$ & $0.32906(6)$ & $0.0163(3)$ \\
H1SA & $0.363(5)$ & $0.650(2)$ & $0.3269(9)$ & $0.020^{*}$ \\
H1SB & $0.059(5)$ & $0.630(2)$ & $0.3289(9)$ & $0.020^{*}$ \\
& & & &
\end{tabular}

Atomic displacement parameters $\left(\AA^{2}\right)$

\begin{tabular}{lllllll}
\hline & $U^{11}$ & $U^{22}$ & $U^{33}$ & $U^{12}$ & $U^{13}$ & $U^{23}$ \\
\hline C1 & $0.0117(8)$ & $0.0117(8)$ & $0.0118(8)$ & $-0.0003(6)$ & $0.0012(6)$ & $-0.0011(6)$ \\
N2 & $0.0141(8)$ & $0.0088(7)$ & $0.0131(7)$ & $-0.0006(6)$ & $-0.0040(6)$ & $0.0023(6)$ \\
C3 & $0.0116(9)$ & $0.0105(8)$ & $0.0110(8)$ & $0.0009(6)$ & $-0.0005(6)$ & $-0.0011(6)$ \\
N4 & $0.0140(8)$ & $0.0095(7)$ & $0.0141(7)$ & $-0.0014(6)$ & $-0.0023(6)$ & $0.0011(5)$ \\
C5 & $0.0123(9)$ & $0.0104(7)$ & $0.0148(9)$ & $-0.0017(6)$ & $-0.0024(7)$ & $0.0005(6)$ \\
N6 & $0.0161(8)$ & $0.0099(7)$ & $0.0144(8)$ & $-0.0012(6)$ & $-0.0048(6)$ & $0.0016(6)$ \\
N7 & $0.0184(8)$ & $0.0109(7)$ & $0.0155(8)$ & $-0.0025(6)$ & $-0.0063(6)$ & $0.0025(6)$ \\
N8 & $0.0148(8)$ & $0.0108(7)$ & $0.0152(8)$ & $-0.0039(6)$ & $-0.0036(6)$ & $0.0036(6)$
\end{tabular}




\begin{tabular}{lllllll} 
N9 & $0.0257(9)$ & $0.0117(7)$ & $0.0222(9)$ & $-0.0067(7)$ & $-0.0141(7)$ & $0.0064(6)$ \\
O11 & $0.0150(7)$ & $0.0108(6)$ & $0.0208(7)$ & $-0.0009(5)$ & $-0.0040(5)$ & $0.0034(5)$ \\
C12 & $0.0135(9)$ & $0.0115(8)$ & $0.0136(9)$ & $-0.0024(7)$ & $-0.0013(7)$ & $0.0022(6)$ \\
O13 & $0.0269(8)$ & $0.0163(6)$ & $0.0231(7)$ & $-0.0048(6)$ & $-0.0135(6)$ & $0.0034(5)$ \\
C14 & $0.0197(10)$ & $0.0113(8)$ & $0.0174(9)$ & $-0.0010(7)$ & $-0.0069(7)$ & $-0.0001(7)$ \\
C15 & $0.0164(9)$ & $0.0128(8)$ & $0.0174(9)$ & $0.0025(7)$ & $-0.0027(7)$ & $0.0037(7)$ \\
N16 & $0.0273(10)$ & $0.0198(8)$ & $0.0235(9)$ & $-0.0010(7)$ & $-0.0104(7)$ & $0.0012(7)$ \\
O1S & $0.0129(7)$ & $0.0126(6)$ & $0.0232(7)$ & $-0.0003(5)$ & $-0.0031(5)$ & $0.0052(5)$ \\
\hline
\end{tabular}

Geometric parameters $\left(\AA,{ }^{\circ}\right)$

\begin{tabular}{|c|c|c|c|}
\hline $\mathrm{C} 1-\mathrm{N} 6$ & $1.322(2)$ & $\mathrm{N} 8-\mathrm{H} 8 \mathrm{~B}$ & $0.88(2)$ \\
\hline $\mathrm{C} 1-\mathrm{N} 7$ & $1.329(2)$ & N9-H9A & $0.90(2)$ \\
\hline $\mathrm{C} 1-\mathrm{N} 2$ & $1.368(2)$ & N9-H9B & $0.90(2)$ \\
\hline $\mathrm{N} 2-\mathrm{C} 3$ & $1.365(2)$ & $\mathrm{O} 11-\mathrm{C} 12$ & $1.268(2)$ \\
\hline $\mathrm{N} 2-\mathrm{H} 2$ & $0.90(2)$ & $\mathrm{C} 12-\mathrm{O} 13$ & $1.238(2)$ \\
\hline $\mathrm{C} 3-\mathrm{N} 4$ & $1.326(2)$ & $\mathrm{C} 12-\mathrm{C} 14$ & $1.536(2)$ \\
\hline $\mathrm{C} 3-\mathrm{N} 8$ & $1.326(2)$ & $\mathrm{C} 14-\mathrm{C} 15$ & $1.458(2)$ \\
\hline $\mathrm{N} 4-\mathrm{C} 5$ & $1.361(2)$ & $\mathrm{C} 14-\mathrm{H} 14 \mathrm{~A}$ & 0.9900 \\
\hline $\mathrm{C} 5-\mathrm{N} 9$ & $1.317(2)$ & C14-H14B & 0.9900 \\
\hline $\mathrm{C} 5-\mathrm{N} 6$ & $1.353(2)$ & $\mathrm{C} 15-\mathrm{N} 16$ & $1.145(2)$ \\
\hline N7-H7A & $0.89(2)$ & $\mathrm{O} 1 \mathrm{~S}-\mathrm{H} 1 \mathrm{SA}$ & $0.93(2)$ \\
\hline N7-H7B & $0.92(2)$ & $\mathrm{O} 1 \mathrm{~S}-\mathrm{H} 1 \mathrm{SB}$ & $0.87(2)$ \\
\hline $\mathrm{N} 8-\mathrm{H} 8 \mathrm{~A}$ & $0.91(2)$ & & \\
\hline $\mathrm{N} 6-\mathrm{C} 1-\mathrm{N} 7$ & $120.78(16)$ & $\mathrm{C} 3-\mathrm{N} 8-\mathrm{H} 8 \mathrm{~A}$ & $121.0(13)$ \\
\hline $\mathrm{N} 6-\mathrm{C} 1-\mathrm{N} 2$ & $121.44(15)$ & $\mathrm{C} 3-\mathrm{N} 8-\mathrm{H} 8 \mathrm{~B}$ & $117.0(14)$ \\
\hline $\mathrm{N} 7-\mathrm{C} 1-\mathrm{N} 2$ & $117.77(15)$ & $\mathrm{H} 8 \mathrm{~A}-\mathrm{N} 8-\mathrm{H} 8 \mathrm{~B}$ & $121.5(19)$ \\
\hline $\mathrm{C} 3-\mathrm{N} 2-\mathrm{C} 1$ & $119.08(14)$ & $\mathrm{C} 5-\mathrm{N} 9-\mathrm{H} 9 \mathrm{~A}$ & $122.0(14)$ \\
\hline $\mathrm{C} 3-\mathrm{N} 2-\mathrm{H} 2$ & $120.1(13)$ & $\mathrm{C} 5-\mathrm{N} 9-\mathrm{H} 9 \mathrm{~B}$ & $121.5(14)$ \\
\hline $\mathrm{C} 1-\mathrm{N} 2-\mathrm{H} 2$ & $120.8(13)$ & H9A-N9-H9B & $116(2)$ \\
\hline $\mathrm{N} 4-\mathrm{C} 3-\mathrm{N} 8$ & $119.86(16)$ & $\mathrm{O} 13-\mathrm{C} 12-\mathrm{O} 11$ & $126.04(16)$ \\
\hline $\mathrm{N} 4-\mathrm{C} 3-\mathrm{N} 2$ & $121.68(15)$ & $\mathrm{O} 13-\mathrm{C} 12-\mathrm{C} 14$ & $116.09(15)$ \\
\hline $\mathrm{N} 8-\mathrm{C} 3-\mathrm{N} 2$ & $118.45(15)$ & $\mathrm{O} 11-\mathrm{C} 12-\mathrm{C} 14$ & $117.87(15)$ \\
\hline $\mathrm{C} 3-\mathrm{N} 4-\mathrm{C} 5$ & $115.76(15)$ & $\mathrm{C} 15-\mathrm{C} 14-\mathrm{C} 12$ & $114.17(14)$ \\
\hline $\mathrm{N} 9-\mathrm{C} 5-\mathrm{N} 6$ & $117.29(15)$ & $\mathrm{C} 15-\mathrm{C} 14-\mathrm{H} 14 \mathrm{~A}$ & 108.7 \\
\hline $\mathrm{N} 9-\mathrm{C} 5-\mathrm{N} 4$ & $117.30(16)$ & $\mathrm{C} 12-\mathrm{C} 14-\mathrm{H} 14 \mathrm{~A}$ & 108.7 \\
\hline $\mathrm{N} 6-\mathrm{C} 5-\mathrm{N} 4$ & $125.42(15)$ & $\mathrm{C} 15-\mathrm{C} 14-\mathrm{H} 14 \mathrm{~B}$ & 108.7 \\
\hline $\mathrm{C} 1-\mathrm{N} 6-\mathrm{C} 5$ & $116.32(15)$ & $\mathrm{C} 12-\mathrm{C} 14-\mathrm{H} 14 \mathrm{~B}$ & 108.7 \\
\hline $\mathrm{C} 1-\mathrm{N} 7-\mathrm{H} 7 \mathrm{~A}$ & $116.4(13)$ & $\mathrm{H} 14 \mathrm{~A}-\mathrm{C} 14-\mathrm{H} 14 \mathrm{~B}$ & 107.6 \\
\hline $\mathrm{C} 1-\mathrm{N} 7-\mathrm{H} 7 \mathrm{~B}$ & $121.4(13)$ & $\mathrm{N} 16-\mathrm{C} 15-\mathrm{C} 14$ & $179.30(19)$ \\
\hline H7A-N7-H7B & $122.1(19)$ & $\mathrm{H} 1 \mathrm{SA}-\mathrm{O} 1 \mathrm{~S}-\mathrm{H} 1 \mathrm{SB}$ & $106.4(19)$ \\
\hline $\mathrm{N} 6-\mathrm{C} 1-\mathrm{N} 2-\mathrm{C} 3$ & $4.1(2)$ & $\mathrm{C} 3-\mathrm{N} 4-\mathrm{C} 5-\mathrm{N} 6$ & $2.5(3)$ \\
\hline $\mathrm{N} 7-\mathrm{C} 1-\mathrm{N} 2-\mathrm{C} 3$ & $-176.16(16)$ & $\mathrm{N} 7-\mathrm{C} 1-\mathrm{N} 6-\mathrm{C} 5$ & $-179.16(16)$ \\
\hline $\mathrm{C} 1-\mathrm{N} 2-\mathrm{C} 3-\mathrm{N} 4$ & $-5.8(2)$ & $\mathrm{N} 2-\mathrm{C} 1-\mathrm{N} 6-\mathrm{C} 5$ & $0.6(2)$ \\
\hline $\mathrm{C} 1-\mathrm{N} 2-\mathrm{C} 3-\mathrm{N} 8$ & $174.98(16)$ & $\mathrm{N} 9-\mathrm{C} 5-\mathrm{N} 6-\mathrm{C} 1$ & $176.40(17)$ \\
\hline $\mathrm{N} 8-\mathrm{C} 3-\mathrm{N} 4-\mathrm{C} 5$ & $-178.19(16)$ & $\mathrm{N} 4-\mathrm{C} 5-\mathrm{N} 6-\mathrm{C} 1$ & $-4.1(3)$ \\
\hline
\end{tabular}


supporting information

\begin{tabular}{llll}
$\mathrm{N} 2-\mathrm{C} 3-\mathrm{N} 4-\mathrm{C} 5$ & $2.6(2)$ & $\mathrm{O} 13-\mathrm{C} 12-\mathrm{C} 14-\mathrm{C} 15$ & $174.83(17)$ \\
$\mathrm{C} 3-\mathrm{N} 4-\mathrm{C} 5-\mathrm{N} 9$ & $-177.98(17)$ & $\mathrm{O} 11-\mathrm{C} 12-\mathrm{C} 14-\mathrm{C} 15$ & $-4.3(3)$ \\
\hline
\end{tabular}

Hydrogen-bond geometry $\left(A,{ }^{\circ}\right)$

\begin{tabular}{lllll}
\hline$D-\mathrm{H} \cdots A$ & $D-\mathrm{H}$ & $\mathrm{H} \cdots A$ & $D \cdots A$ & $D-\mathrm{H} \cdots A$ \\
\hline $\mathrm{N} 2-\mathrm{H} 2 \cdots \mathrm{O} 1 S$ & $0.90(2)$ & $1.81(2)$ & $2.7067(19)$ & $176.4(19)$ \\
$\mathrm{N} 7-\mathrm{H} 7 A \cdots \mathrm{O} 13^{\mathrm{i}}$ & $0.89(2)$ & $2.00(2)$ & $2.881(2)$ & $168(2)$ \\
$\mathrm{N} 7-\mathrm{H} 7 B \cdots \mathrm{N} 16^{\mathrm{ii}}$ & $0.92(2)$ & $2.13(2)$ & $3.001(2)$ & $155.6(18)$ \\
$\mathrm{N} 8-\mathrm{H} 8 A \cdots \mathrm{O} 11$ & $0.91(2)$ & $2.01(2)$ & $2.891(2)$ & $164.6(19)$ \\
$\mathrm{N} 8-\mathrm{H} 8 B \cdots \mathrm{N} 44^{\mathrm{iii}}$ & $0.88(2)$ & $2.07(2)$ & $2.952(2)$ & $176(2)$ \\
$\mathrm{N} 9-\mathrm{H} 9 A \cdots \mathrm{O} 13^{\mathrm{iii}}$ & $0.90(2)$ & $2.08(2)$ & $2.792(2)$ & $135.7(19)$ \\
$\mathrm{N} 9-\mathrm{H} 9 B \cdots \mathrm{N} 66^{\mathrm{iv}}$ & $0.90(2)$ & $2.08(2)$ & $2.980(2)$ & $174(2)$ \\
$\mathrm{C} 14-\mathrm{H} 14 B \cdots \mathrm{O} 1 S^{\mathrm{v}}$ & 0.99 & 2.46 & $3.233(2)$ & 134 \\
$\mathrm{O} 1 S-\mathrm{H} 1 S A \cdots \mathrm{O} 11$ & $0.93(2)$ & $1.78(2)$ & $2.6860(19)$ & $163.3(19)$ \\
$\mathrm{O} 1 S-\mathrm{H} 1 S B \cdots \mathrm{O} 11^{\mathrm{vi}}$ & $0.87(2)$ & $1.97(2)$ & $2.8351(19)$ & $178(2)$ \\
& & & &
\end{tabular}

Symmetry codes: (i) $x-1, y-1, z$; (ii) $-x, y-1 / 2,-z+1 / 2$; (iii) $-x+2,-y+1,-z+1$; (iv) $-x+1,-y,-z+1$; (v) $-x+1, y+1 / 2,-z+1 / 2$; (vi) $x-1, y, z$. 